\title{
In vitro Simulation of Periodontal Ligament in Fatigue Testing of Dental Crowns
}

\author{
Noor Nawafleh ${ }^{1} \quad$ Abdel Raheem Bibars ${ }^{1} \quad$ Shareen Elshiyab ${ }^{1} \quad$ Yasmeen Janzeer $^{1}$ \\ ${ }^{1}$ Department of Applied Dental Sciences, Faculty of Applied Medical \\ Sciences, Jordan University of Science and Technology, Irbid, Jordan \\ Address for correspondence Noor Nawafleh, BSc, MDent Tech, \\ PhD, Faculty of Applied Medical Sciences, Jordan University of \\ Science and Technology, Irbid 22110, Jordan \\ (e-mail: nanawafleh@just.edu.jo).
}

Eur J Dent:2020;14:380-385

\begin{abstract}
Objective Fatigue testing of restorative material has been appreciated as an appropriate method to evaluate dental restorations. This study aims to investigate the influence of periodontal ligament (PDL) simulation on fatigue and fracture tests results of zirconia crowns.

Materials and Methods A standard tooth preparation for all ceramic zirconia crown was made on a typodont mandibular molar. The prepared master die was duplicated using epoxy resin to produce 40 replicas. PDL simulation was made by surrounding the root of 20 dies with a $0.3-\mathrm{mm}$ thick silicon layer. The other 20 specimens had no PDL simulation. Zirconia crowns were fabricated using computer-aided design/computer-aided manufacturing technology and cemented to the epoxy resin dies. Ten crowns from each group were subject to chewing simulation with simultaneous thermocycling $\left(5-55^{\circ} \mathrm{C}\right)$. All specimens were then loaded until failure in universal testing machine.

Statistical Analysis Statistical analysis was conducted using SPSS software. ShapiroWilk test confirmed the normal distribution of data. Descriptive statistic was performed and differences between the groups were analyzed using paired samples $t$-test.

Results All fatigued crowns survived chewing simulation; no failure was observed after finishing simulation. The highest mean fracture load recorded was 3,987 $\pm 400 \mathrm{~N}$

Keywords

- chewing simulation

- fatigue

- fracture resistance

- periodontal ligament

- zirconia crown for the no fatigue/no periodontal simulation group. Comparing the mean fracture load of the two groups with periodontal simulation and the two groups with no periodontal simulation showed no statistically significant difference $(p>0.5)$.

Conclusion Considering the testing set-up applied in this study, simulating PDL using resilient materials does not affect the in vitro survival and fracture resistance of zirconia crowns.
\end{abstract}

\section{Introduction}

Undeniably, laboratory studies play a major role in the development of dental restorative materials. In vitro studies, whether simplified or with complex approaches add to our understanding of the properties and mechanical behavior of dental materials and restorations. ${ }^{1,2}$ Two different approaches of in vitro studies can be identified in the literature; studies aim at measuring physical and mechanical properties, and studies aim to simulate clinical behavior. ${ }^{3}$ Although the first approach is important in providing information about material properties, estimating failure risk and comparing material variants, ${ }^{4}$ it is known clinically that failures of all-ceramic restorations are a complex process which occur mostly as a result of fatigue stresses in challenging oral conditions. ${ }^{5,6}$ Therefore, the need for laboratory simulations of clinical situation should not be ignored. Chewing simulation on dental restorations are considered a promising and favorable approach to estimate the clinical performance of dental restorations in vitro. ${ }^{1,6-9}$ It can provide the first meaningful 
estimation of the material's performance in the shape of a dental restoration ${ }^{10}$ and therefore experts suggest to consider strong restrictions on publishing papers using only simple bend tests and load-to-failure methods as they are not representative and have limited clinical relevance. ${ }^{3}$

Laboratory studies which incorporate fatigue testing have been advocated to have a high-translational meaning.,11 Many articles have been published in the past few years providing valuable input in understanding this topic and guiding new research. ${ }^{1,10,12,13}$ Nevertheless, reviewing recent publications shows considerable variations in fatigue testing methodologies, which make the comparability of results a serious challenge. Fatigue testing studies vary in many aspects such as the number and frequency of cycles, abutment and antagonist materials, periodontal ligament (PDL) simulation, and sometimes thermocycling protocols., ${ }^{4,10,14,15}$ Therefore, identifying the influence of each variable on testing and subsequent results can be of great importance in guiding future research. In addition, mechanical properties and failure mechanism of each material should be considered; different materials might need different testing set-ups to be more representative to the clinical situation.

PDL simulation is one of the experimental variables that has been identified as an influential factor in fatigue testing. ${ }^{10,14,16,17}$ Many studies applied PDL simulation during testing using both linear ${ }^{18-20}$ and dynamic compressive loads. ${ }^{21-}$ ${ }^{23}$ Other studies, however, did not include this simulation in study design. ${ }^{8,24,25}$ Naumann et $\mathrm{al}^{4}$ reviewed the designs of 69 in vitro studies investigated the fracture resistance of postendodontic restorations. They found that $50 \%$ of the fatigue testing studies and $72 \%$ of the studies that applied single load to fracture test did not incorporate PDL layer in their specimen's structure. ${ }^{4}$ Another review ${ }^{14}$ focused on the fatigue testing parameters applied in testing lithium disilicate restoration found that only 7 studies (out of 19 studies) applied PDL simulation, mostly on fibrin degradation products (FDPs) specimens.

Materials like polyether and polysiloxane among others have been used to substitute for PDL during laboratory simulations. However, the differences in mechanical and physical properties, layer thicknesses, and the techniques involved in the production of the artificial PDL in vitro have been found to affect the accuracy and reproducibility of the simulated PDL layer. ${ }^{26}$ This makes the success of such simulation questionable. Therefore, this study investigates the influence of artificial PDL on the results of fatigue survival and fracture load of zirconia crowns. The formulated hypotheses were: PDL simulation has no effect on in vitro fatigue survival (1.2 million cycles) and fracture load results of fatigued and unfatigued zirconia crowns.

\section{Materials and Methods}

\section{Specimens' Preparation}

A standard tooth preparation for all-ceramic zirconia crown was made on a typodont mandibular first molar (Nissin Dental Products Inc.; Kyoto, Japan) with an occlusal reduction of $1.5 \mathrm{~mm}$, a proximal/axial wall reduction of $1.0 \mathrm{~mm}$, and $0.5 \mathrm{~mm}$ finish-line depth. The preparation depth was controlled using silicone index of unprepared tooth to achieve the required tooth reduction. Total 40 impressions of the master die were made from 3M ESPE impression material (St. Paul, Minnesota, United States) and poured with epoxy resin material (Exakto-Form, Bredent, Germany) to produce 40 replicas of the prepared master dies.

The master cast was scanned using a desktop scanner (Ceramill Map 400; Amann Girrbach, Germany) and saved in stereolithography (STL) format. STL data were then imported into dental CAD software (Design software; Amann Girrbach, Germany) to design the molar crowns. Crowns were fabricated from CeramillZolid HT (CER; Amann Girrbach, Germany) using five-axis milling machine (Ceramill Motion 2; Amann Girrbach AG, Germany). Crowns were cemented to the epoxy resin dies using Multilink (IvoclarVivadent, Shaan, Liechtenstein) according to the manufacturer's instructions and stored in water until testing.

The master die was fixed in a standardized position in the chewing simulator's specimen cup using a thin mix of cold autopolymerizing acrylic resin (Megadental; Büdingen, Germany) covering the die up to $2.0 \mathrm{~mm}$ away from the finish line. In order to simulate the PDLs in $50 \%$ of the specimens, a 0.3-mm thick wax layer (Schuler-Dental; Ulm, Germany) was added to the roots before pouring the acrylic resin mix. The wax was then dewaxed and substituted with light body elastomeric impression material (3M ESPE; Minnesota, United States). This silicon layer is supposed to allow for a minimal movement of the dies in its acrylic resin socket similar to the effect of the periodontal membrane around natural teeth.

\section{Chewing Simulation}

Crowns were divided according to the inclusion PDL simulation and fatigue application into four groups of ten specimens:

- Fatigue/PDL

- Fatigue/no PDL

- No fatigue/PDL simulation

- No fatigue/no PDL simulation

Two groups were subjected to thermal mechanical loading in chewing simulator (CS-4.8; SD Mechatronik, FeldkirchenWesterham, Germany) for 1.2 million cycles. Loading force was set at $100 \mathrm{~N}$ with a loading frequency of $1.2 \mathrm{~Hz}$. Ceramic spherical indenter with $6 \mathrm{~mm}$ diameter was used to load the specimens during chewing simulation. In each loading cycle, the indenter was hitting the specimen at $0.5 \mathrm{~mm}$ lingual to the distobuccal cusp tip, applied the load, slided $0.3 \mathrm{~mm}$ lingually, and left the specimen with a vertical distance of $2 \mathrm{~mm}$ (-Fig. 1). The precise starting point of the indenter at the distobuccal cusp incline for all specimens was identified using articulating paper and guaranteed to be identical for all specimens by the standardized position of the crowns in the specimen's cup ( $\mathbf{- F i g}$. 1). Thermocycling between 5 and $55^{\circ} \mathrm{C}$ in distilled water (dwell time: 60 seconds, drain time: 15 seconds) was run all through the testing 


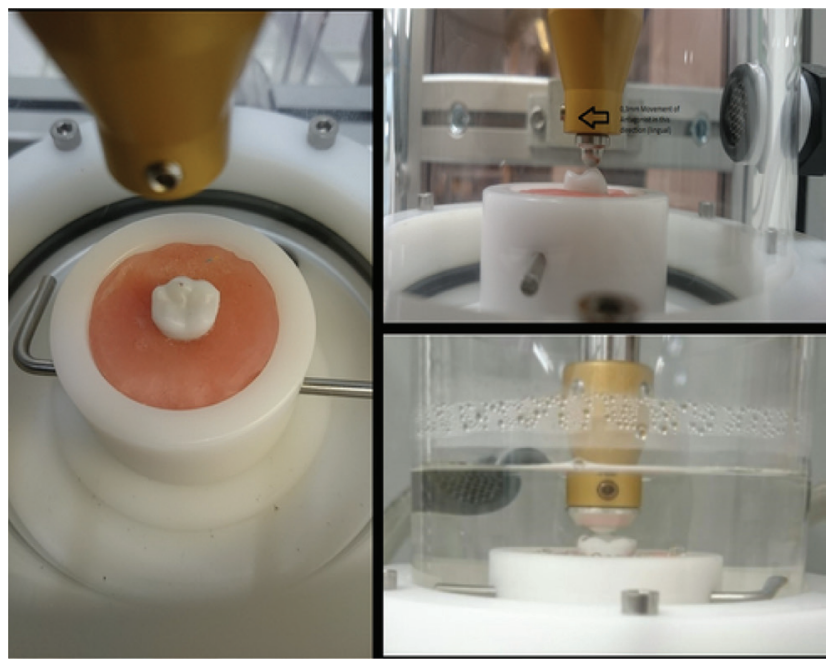

Fig. 1 Chewing simulation with a simultaneous thermocycling of the zirconia crowns.

using computerized thermocycling unit (SD Mechatronik, Feldkirchen-Westerham, Germany).

\section{Single Load to Fracture}

After finishing 1.2 million cycles, fatigued crowns were inspected for cracks or fractures using a stereomicroscope at $20 \times$ (M125; Leica Microsystems, Wetzlar, Germany). All crowns (fatigued and unfatigued) were loaded to failure in universal testing machine (Jinan Testing Equipment IE Corporation, China). In order to place all specimens in the same reproducible position in the universal testing machine, a specially designed jig ( - Fig. 2 ) was manufactured in accordance with the specimen's cup of the chewing simulator and the loading table of the universal testing machine. The specimens were fixed in place to achieve tripod contact between the crown and the indenter (contact points were the triangular ridges of the distobuccal cusp and the two palatal cusps). An 8-mm diameter stainless steel indenter was used to apply the load vertically with a crosshead speed of $1 \mathrm{~mm} / \mathrm{min}$ until failure. Fracture load for each crown was recorded in Newton.

Statistical analysis was conducted using SPSS software (SPSS, Chicago, Illinois, United States). Shapiro-Wilk test confirmed the normal distribution of data. Descriptive statistics (mean and standard deviation) was performed, and differences between the groups were analyzed using paired samples $t$-test. One-way ANOVA was not used because the main aim of this study was to compare the two experimental groups with corresponding control groups (unfatigued groups) to identify the influence of adding the PDL simulation. For all statistical analyses, the level of significance was set at $95 \%$.

\section{Results}

All fatigued crowns survived 1.2 million loading cycles with simultaneous thermocycling $\left(5-55^{\circ} \mathrm{C}\right)$ resulting in $100 \%$ survival; no cracks or fractures were observed after the test completion. The highest mean fracture load recorded was $3,987 \mathrm{~N}$ for the no fatigue/no PDL simulation group, and the

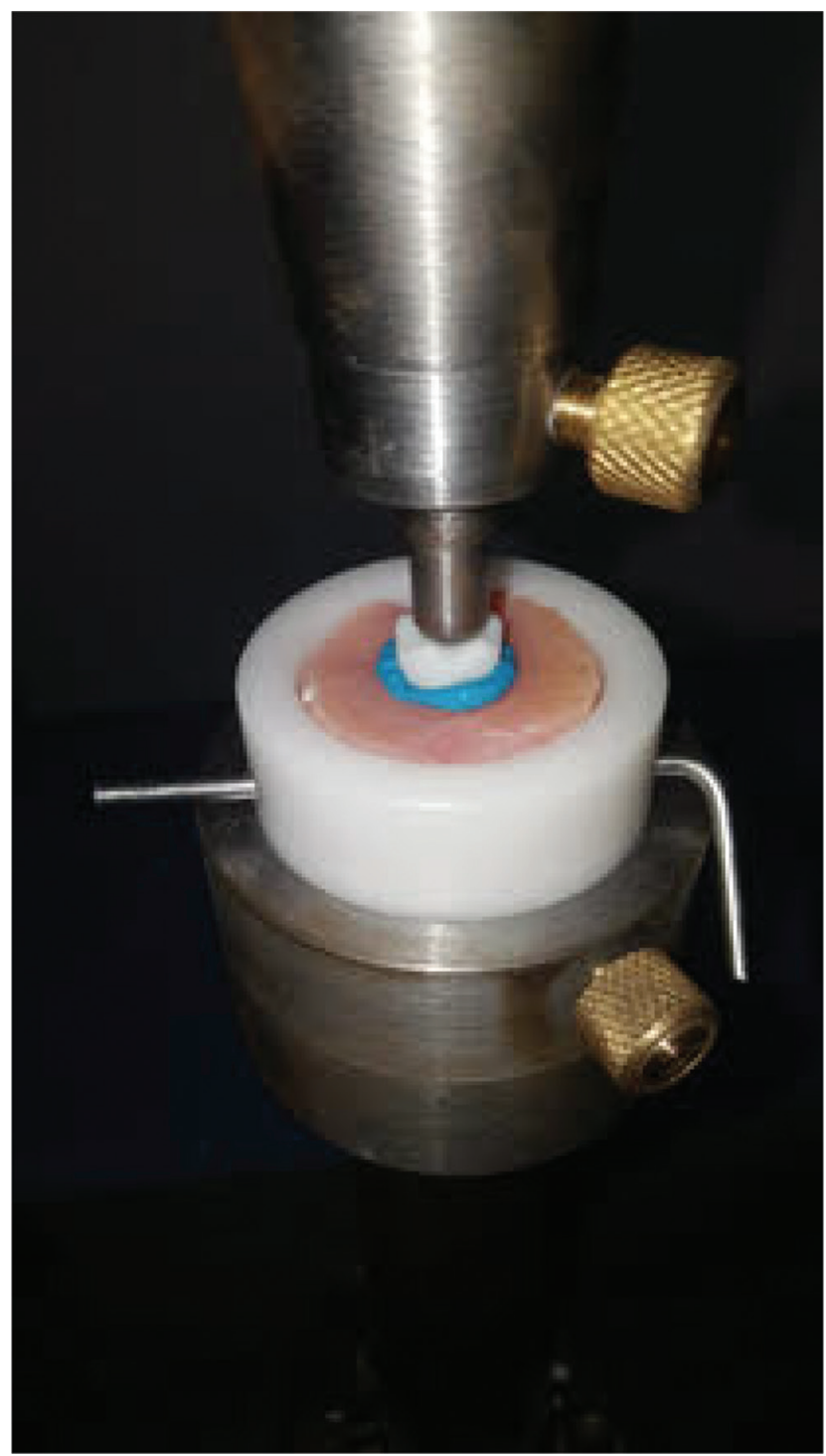

Fig. 2 Single load to fracture test in universal testing machine with specially designed jig into hold the specimens in its standardized position in the chewing simulator specimen's cup.

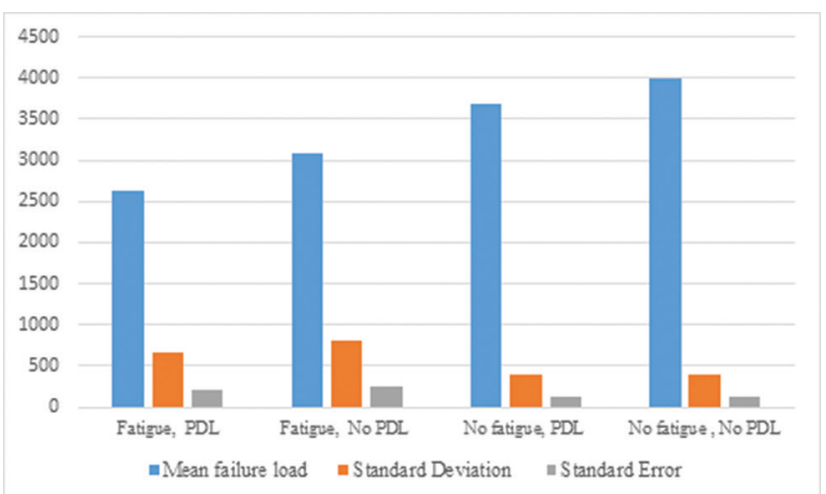

Fig. 3 Bar graph shows results (in Newtons) of mean fracture load, standard deviation, and standard error of fatigued and unfatigued groups.

lowest mean fracture load was 2,635 $\mathrm{N}$ for the Fatigue/PDL. The results of the mean fracture load and standard deviation for the four groups are shown in - Figure 3. Comparing the 
mean fracture load of the unfatigued groups shows no statistically significant difference $(p>0.5)$. Similarly, the difference in the mean fracture load between the two fatigued groups was not statistically significant $(p>0.5)$.

\section{Discussion}

Assessment of experimental model that include a simulation of PDL in comparison to a model without this simulation has appeared in few previous studies..$^{10,17,27,28}$ These studies tested the influence of PDL simulation on fracture resistance of natural teeth ${ }^{27,28}$ and FDPs. ${ }^{10,17}$ To the best of our knowledge, searching the literature did not reveal any study testing the influence of the PDL simulation on the in vitro fracture resistance of crown restorations after chewing simulation. Therefore, direct comparison with previous similar studies is not possible.

This study found no significant difference in fracture load between groups with and without PDL simulation regardless of fatigue application. This disagrees with Rosentritt et $\mathrm{al}^{10}$ who used FDPs specimens and found that omitting the artificial periodontium during thermal mechanical loading caused fracture results almost twice the fracture load recorded with PDL simulation. Based on their results, Rosentritt et al ${ }^{10}$ recommended the application of artificial periodontium during fatigue and single load to fracture testing. In another study, ${ }^{17}$ also used FDPs specimens, the fracture resistance was significantly reduced when artificial periodontium was applied during thermal mechanical cycling and single load to fracture test. The difference in specimens' structure (crown or FDP) can explain this disagreement. The mechanical failure in FDPs, which have more complex geometry compared to single crowns, mostly occur as a result of tensile stresses at the tissue side of the connectors, and therefore, the PDL simulation can play a significant role. ${ }^{29}$

The results of this study can be explained by the design of the fracture test. In the majority of fatigue testing studies, cyclic loading is applied for a specific number of cycles and followed by fracture test, which lead to the catastrophic failure of specimens. Similarly, all crowns in this study survived fatigue and failed as a result of single load to fracture test. In this test, the indenter gradually applies a load until failure, which compresses the resilient layer representing the PDL between two solid fixtures (acrylic socket and epoxy resin die). At some point, with the continuously increased load, the elastomer material is compressed past its elastic limit and then its cushioning effect is no longer existing. ${ }^{30}$ When this elastomeric layer become totally squeezed that might occur at early stages of the test, the load applied by the indenter only influence the specimen until it fails, ${ }^{28}$ which result in no significant differences between the groups with and without PDL simulation.

On the other hand, the cushioning effect of the PDL simulation might be more influential during fatigue testing because of the relatively low load (100 N) compared to the load applied during fracture test. This means that in longterm simulations at normal physiologic occlusal load or if the specimens fatigued until failure, the elastic property of the
PDL simulation material can last longer and might influence the in vitro survival of the restoration. However, according to the results of this study, up to 1.2 million cycles at $100 \mathrm{~N}$ the PDL simulation has no significant effect on the survival and postfatigue fracture load results.

In a similar study using natural teeth, Marchionatti et $\mathrm{a}^{28}$ studied the influence of PDL simulation on fracture resistance of roots restored with fiber posts. They noticed from the relationship between load and deformation occurs in the specimen during fracture test that the groups included PDL simulation presented premature peaks before failure. These peaks indicate the accumulation of the specimen in the artificial socket by the simulated PDL. ${ }^{28}$ However, specimens in the groups with no PDL simulation do not show these premature peaks before failure threshold. - Figure 4 shows the graphics of the relationships for the four groups tested in the current study as generated by the universal testing machine, which agree with Marchionatti et $\mathrm{al}^{28}$ observations.

Investigating the influence of PDL simulation on the results of in vitro fatigue testing through a real laboratory testing is very rare in the literature. ${ }^{10,17}$ However, it has been emphasized in many finite element (FE) studies. According to FE studies, including PDL in the simulation modifies the distribution of stress and strain ${ }^{31}$ in the computed models. Therefore, it has been advocated that PDL simulation is a fundamental step in FE modelling, ${ }^{31}$ and it should be included in experimental models. ${ }^{17}$ However, in a critical review of the literature about the analytically determined mechanical properties of PDL and its modelling strategies in FE studies, ${ }^{32}$ the authors found that the acknowledgement of great complexity of the biomechanical behavior of the PDL material, but using basic and simplified models in the real study are pervasive throughout the literature. ${ }^{32}$ They also found a considerable inconsistency and discrepancy of numerical approaches used in characterizing PDL behavior. ${ }^{32}$ Despite the substantial developments that have been made in recent FE packages, the results of FE simulations must be carefully studied before they can be applied. ${ }^{33}$

Brosh et $\mathrm{a}^{16}$ analyzed the suitability of resilient materials used in dental research to simulate the viscoelastic behavior of the PDLand compared some commonly used materials interms of recovery and tensile relaxation tests. They reported significant difference in elastic modulus and relaxation behavior

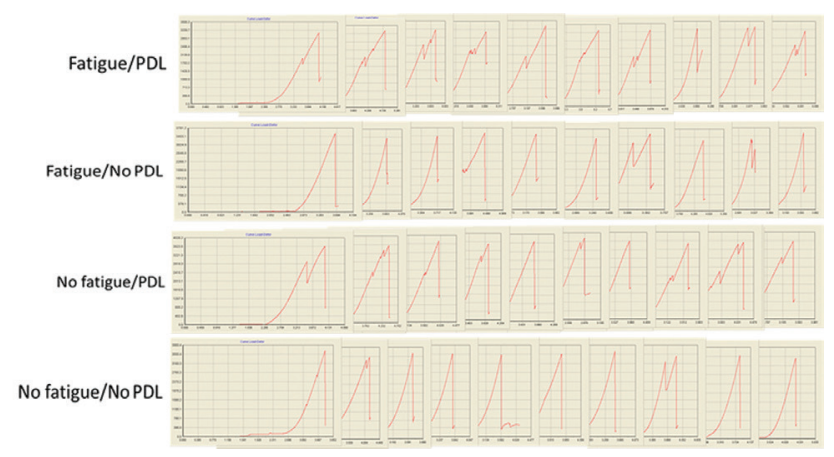

Fig. 4 Graphics of the relationships for the four groups tested in the current study as generated by the universal testing machine. PDL, periodontal ligament. 
between different materials usually used as a substitute for PDL. ${ }^{16}$ PDL simulation was avoided in some studies because it adds complexity to the design ${ }^{34}$ and can cause dislodgement of the tooth during testing, which influences the final assessment of the material under investigation. Heintze et $\mathrm{al}^{29}$ argued that in case of testing crown specimens, the application of artificial periodontium would decrease the axial force as the abutment moves in an unstandardizable elastic layer, which will more likely results in an uncontrolled and unstandardized mobility. ${ }^{29}$ In another study, Heintze et al ${ }^{35}$ justified not using artificial periodontium in case of FPDs specimens by the variation in the thickness of the silicone layer (300 and $700 \mu \mathrm{m}$ ) that they found from their own experience. This variation makes it unstandardized and the subsequent mobility of the abutment teeth during loading would be uncontrolled and unstandardized. ${ }^{35}$

In a systematic review of the experimental variables involved in fatigue testing of restorative materials, it has been advised that simulation of all intraoral conditions including PDL should be as close as possible ${ }^{14}$ because the influence of each factor on the results has not been confirmed sufficiently in the literature. However, up to date, the simulation of PDL in vitro lacks standardization in terms of materials, techniques, and dimensions. ${ }^{26}$ Many researches stressed on confirming the importance of including a viscoelastic layer to simulate tooth mobility allowed by the PDL. Yet, evidence of achieving a reproducible layer of the viscoelastic material that can produce a standardized and clinically relevant movement of the abutment tooth during testing has never been reported. Therefore, the uncertainty of the successful simulation in addition to the complexity added to the design may suggest eliminating this variable from the testing setup, especially if it has no significant influence on the results.

\section{Conclusion}

Within the limitations of this study, it can be concluded that the simulation PDL using resilient materials does not affect the in vitro survival and fracture test results of single zirconia crowns. Therefore, considering the testing set-up applied in this study, PDL simulation-which add more complexity to the specimen's structure-would not be necessary.

Further research is needed to confirm the results of this study on zirconia crowns and other materials, which may show different results.

\section{Conflict of Interest}

None declared.

\section{Acknowledgement}

This work was supported by Jordan University of Science and Technology, Irbid, Jordan.

\section{References}

1 Rosentritt M, Behr M, Preis V. A critical evaluation of fatigue studies for restorative materials in dentistry. Curr Oral Health Rep 2016;3:221-228
2 Alqahtani F, AlAmar M. In vitro comparison of modes of failures among titanium and one-and two-piece zirconia abutment under static load. Eur J Dent 2020;14(1):157-160

3 Kelly JR, Benetti P, Rungruanganunt P, Bona AD. The slippery slope: critical perspectives on in vitro research methodologies. Dent Mater 2012;28(1):41-51

4 Naumann M, Metzdorf G, Fokkinga W, et al. Influence of test parameters on in vitro fracture resistance of post-endodontic restorations: a structured review. J Oral Rehabil 2009;36(4):299-312

5 Denry I, Holloway J. Ceramics for dental applications: a review. Materials (Basel) 2010;3:351-368

6 Heintze SD, Albrecht T, Cavalleri A, Steiner M. A new method to test the fracture probability of all-ceramic crowns with a dual-axis chewing simulator. Dent Mater 2011;27(2):e10-e19

7 Rosentritt M, Siavikis G, Behr M, Kolbeck C, Handel G. Approach for valuating the significance of laboratory simulation. J Dent 2008;36(12):1048-1053

8 Seydler B, Rues S, Müller D, Schmitter M. In vitro fracture load of monolithic lithium disilicate ceramic molar crowns with different wall thicknesses. Clin Oral Investig 2014;18(4):1165-1171

9 Moradi Z, Abbasi M, Khalesi R, Tabatabaei MH, Shahidi Z. Fracture toughness comparison of three indirect composite resins using 4-point flexural strength method. Eur J Dent 2020. Doi:10.1055/s-0040-1708438

10 Rosentritt M, Behr M, Gebhard R, Handel G. Influence of stress simulation parameters on the fracture strength of all-ceramic fixed-partial dentures. Dent Mater 2006;22(2): 176-182

11 Kelly JR. Clinically relevant approach to failure testing of all-ceramic restorations. J Prosthet Dent 1999;81(6):652-661

12 Kelly JR, Cesar PF, Scherrer SS, et al. ADM guidance-ceramics: fatigue principles and testing. Dent Mater 2017;33(11): 1192-1204

13 LohbauerU, Krämer N, Petschelt A, Frankenberger R. Correlation of in vitro fatigue data and in vivo clinical performance of a glassceramic material. Dent Mater 2008;24(1):39-44

14 Nawafleh N, Hatamleh M, Elshiyab S, Mack F. Lithium disilicate restorations fatigue testing parameters: a systematic review. J Prosthodont 2016;25(2):116-126

15 Nawafleh N, Bibars AR, Al Twal E, Öchsner A. Influence of antagonist material on fatigue and fracture resistance of zirconia crowns. Eur J Dent 2020. Doi: 10.1055/s-0040-1708228

16 Brosh T, Porat N, Vardimon AD, Pilo R. Appropriateness of viscoelastic soft materials as in vitro simulators of the periodontal ligament. J Oral Rehabil 2011;38(12):929-939

17 Rosentritt M, Behr M, Scharnagl P, Handel G, Kolbeck C. Influence of resilient support of abutment teeth on fracture resistance of all-ceramic fixed partial dentures: an in vitro study. Int J Prosthodont 2011;24(5):465-468

18 Akkayan B, Gülmez T. Resistance to fracture of endodontically treated teeth restored with different post systems. J Prosthet Dent 2002;87(4):431-437

19 Newman MP, Yaman P, Dennison J, Rafter M, Billy E. Fracture resistance of endodontically treated teeth restored with composite posts. J Prosthet Dent 2003;89(4):360-367

20 Fokkinga WA, Kreulen CM, Le Bell-Rönnlöf AM, Lassila LV, Vallittu PK, Creugers NH. Fracture behavior of structurally compromised non-vital maxillary premolars restored using experimental fiber reinforced composite crowns. Am J Dent 2006;19(6):326-332

21 Attia A, Kern M. Influence of cyclic loading and luting agents on the fracture load of two all-ceramic crown systems. J Prosthet Dent 2004;92(6):551-556

22 Forberger N, Göhring TN. Influence of the type of post and core on in vitro marginal continuity, fracture resistance, and 
fracture mode of lithia disilicate-based all-ceramic crowns. J Prosthet Dent 2008;100(4):264-273

23 Schultheis S, Strub JR, Gerds TA, Guess PC. Monolithic and bi-layer CAD/CAM lithium-disilicate versus metal-ceramic fixed dental prostheses: comparison of fracture loads and failure modes after fatigue. Clin Oral Investig 2013;17(5): $1407-1413$

24 Carvalho AO, Bruzi G, Giannini M, Magne P. Fatigue resistance of CAD/CAM complete crowns with a simplified cementation process. J Prosthet Dent 2014;111(4):310-317

25 Zhao K, Wei Y-R, Pan Y, Zhang X-P, Swain MV, Guess PC. Influence of veneer and cyclic loading on failure behavior of lithium disilicate glass-ceramic molar crowns. Dent Mater 2014;30(2):164-171

26 AlZahrani F, Richards L. Micro-CT evaluation of a novel periodontal ligament simulation technique for dental experimental models. Arch Orofac Sci 2018;13:93-103

27 Soares CJ, Pizi ECG, Fonseca RB, Martins LRM. Influence of root embedment material and periodontal ligament simulation on fracture resistance tests. Braz Oral Res 2005;19(1):11-16

28 Marchionatti AME, Wandscher VF, Broch J, et al. Influence of periodontal ligament simulation on bond strength and fracture resistance of roots restored with fiber posts. J Appl Oral Sci 2014;22(5):450-458
29 Heintze SD, Cavalleri A, Zellweger G, Büchler A, Zappini G. Fracture frequency of all-ceramic crowns during dynamic loading in a chewing simulator using different loading and luting protocols. Dent Mater 2008;24(10):1352-1361

30 Aboushelib MN. Simulation of cumulative damage associated with long term cyclic loading using a multi-level strain accommodating loading protocol. Dent Mater 2013;29(2):252-258

31 Aversa R, Apicella D, Perillo L, et al. Non-linear elastic three-dimensional finite element analysis on the effect of endocrown material rigidity on alveolar bone remodeling process. Dent Mater 2009;25(5):678-690

32 Fill TS, Toogood RW, Major PW, Carey JP. Analytically determined mechanical properties of, and models for the periodontal ligament: critical review of literature. J Biomech 2012;45(1):9-16

33 Meira JB, Jikihara AN, Capetillo P, Roscoe MG, Cattaneo PM, Ballester RY. Finite element analysis in dentistry. Dent Biomater 2018;2:67

34 Hu YH, Pang LC, Hsu CC, Lau YH. Fracture resistance of endodontically treated anterior teeth restored with four post-andcore systems. Quintessence Int 2003;34(5):349-353

35 Heintze SD, Monreal D, Reinhardt M, et al. Fatigue resistance of all-ceramic fixed partial dentures - fatigue tests and finite element analysis. Dent Mater 2018;34(3):494-507 